\title{
Measurement of a surface profile with maximum accuracy using a temporal phase-stepping
}

\author{
J. Surrel, J. Molimard
}

J. Surrel, J. Molimard, "Measurement of a surface profile with maximum accuracy using a temporal phase-stepping," Proc. SPIE 9664, Ninth International Topical Meeting on Education and Training in Optics and Photonics, 96642M (24 October 2005); doi: 10.1117/12.2207711

SPIE Event: Ninth International Topical Meeting on Education and Training in Optics and Photonics, 2005, Marseille, France 


\title{
Ref ETOP051
}

\section{Measurement of a surface profile with maximum accuracy, using a temporal phase- stepping.}

\author{
J. SURREL ${ }^{1}$, J. MOLIMARD $^{2}$
}

${ }^{1}$ ISTASE : Institut Supérieur des Techniques Avancées de Saint Étienne, 21 rue du Docteur Paul Michelon, 42023 Saint Étienne Cédex, FRANCE

${ }^{2}$ EMSE : École des Mines de Saint Étienne, 158 Cours Fauriel, Saint Étienne Cédex, FRANCE

\begin{abstract}
The spreading of optical full-field technique in the world of mechanics needs a strong educational effort at any level: undergraduate, graduate, or continuing education. On that purpose, practicals are very important. In the context of a "Photomechanics summer school" held by CNRS, a practical based on the fringe projection technique has been developed.

Even if the basic principle is very simple, a lot of parameters have to be fixed. The set-up enables students to understand the choices of these parameters in order to obtain the best results and shows a way to characterize the errors.
\end{abstract}

\section{Keywords}

surface profile measurement, spatial phase stepping, temporal phase stepping, phase modulation, contrast, sensitivity, resolution, noise, visibility, calibration,

\section{Summary}

The optical fringe projection technique for a surface profile measurement is very useful because the whole surface can be analysed at once. A reference optical grid is generated with a videoprojector and projected onto the object surface.

For a given optical setup the shape of the fringes depends on the profile of the surface. In our experiment, a software allows the determination of the object shape. To obtain the best results the experimenter has to choose different parameters: the spatial period of the fringes, the contrast of the pattern, whether to use temporal or spatial phase stepping and the value of the phase step. The setup enables students to understand the choices of the parameters and allows the investigation of the errors that have to be avoided.

To process the fringe pattern the computer is equiped with the FrEngine software. The mean

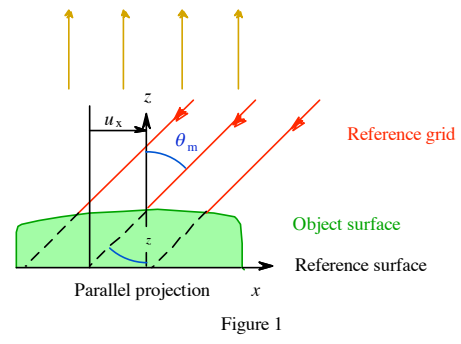
angle $\square_{\mathrm{m}}$ between the video-projector optical axis and the camera one's is about $20^{\circ}$ (figure 1).

When a reference grid generated by the videoprojector is projected onto a reference plane surface $(x, y)$, the intensity field on the plane is described by a periodic function denoted $"$ frg $»$ :

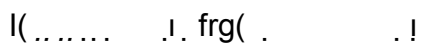

In the simpliest case the periodic function is a cosine function and the intensity at a given pixel contains 3 unknown factors $\square \square$ and $\square$ [Eq. (1)]. So, in order to 
get the phase $\square$, at least three images have to be saved and processed.

When the grid is projected onto an object the fringe pattern is deformed. The shape of the fringe can be described as a phase modulation of the fringe intensity :

$\mathrm{I}(\square, \square \square \square \square \mathrm{I}, \mathrm{frg}(\square \square, \square \square \square \mathrm{I}, \mathrm{frg}(\square \tilde{\square}$

The phase to be collimated, the relationship between the phase and the altitude is :

$\square \square \square \square \tilde{\square}$ ) $\tan (\square \mathrm{m}) z$

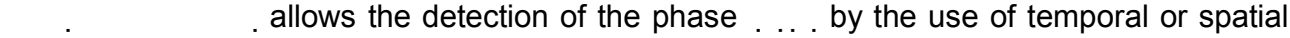
phase-stepping. To obtain the best results the students have to investigate the influence of various parameters :

o using temporal or spatial phase stepping

o the spatial period of the fringes

o the contrast and the fringe profile

o the value of the phase step.

If the object is moving (production chains, biological object) the spatial phase-stepping has to be chosen. On the other hand if one has some time it is better to work with temporal phasestepping to obtain a better spatial resolution. Students have no time enough to investigate the two methods so in the proposed lab work they approach only the temporal phase-stepping.

From Eq (2) the students deduce that the sensitivity $\square \square / \square z$ increases when the angle

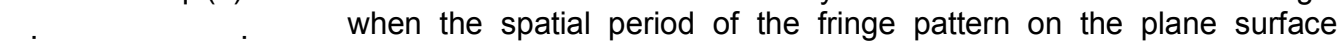
decreases. So they choose a large angle but they have to make sure that there are no shadows. The smallest period is equal to 3 pixels.

To know the detection threshold (or resolution) of the phase measurement, the students measure the noise of the acquisition chain doing the difference between two images of two independent acquisitions of the same reference plane. They should notice that the resolution of the phase measurement decreases when the visibility $\square$ is maximum, and when the mean radiance $A$ of the object surface is important as indicated by the following equation :

$$
\square_{\square}=\sqrt{ }\left(2 \square^{2} / M A \square . . \quad \text {. } \square .\right.
$$

where $\square^{2}$ is the noise power on the intensity and $M$ the number of acquired images. The students have to be careful because the mean radiance $A$ of the object surface has to be maximum but no saturation should occur. Moreover when the reference optical grid is generated with a video-projector they notice that some compromise has to be found between a great visibility and to obtain a cosine function for the intensity on the plane reference.

The last investigated parameter is the $N$ value of the phase step. This number has to be a divisor of the number of pixels of the spatial period of the reference optical grid (here we refer to the videoprojector pixel array). The students should notice two things, the first one is that the number $M$ of images taken to calculate the phase stepping. The second one is that the uncertainty of the phase measurement evaluated by the standard deviation $\square_{\square}$ is inversely proportional to $N$ but that the duration of the experiment becomes important. So, a compromise has to be found. 


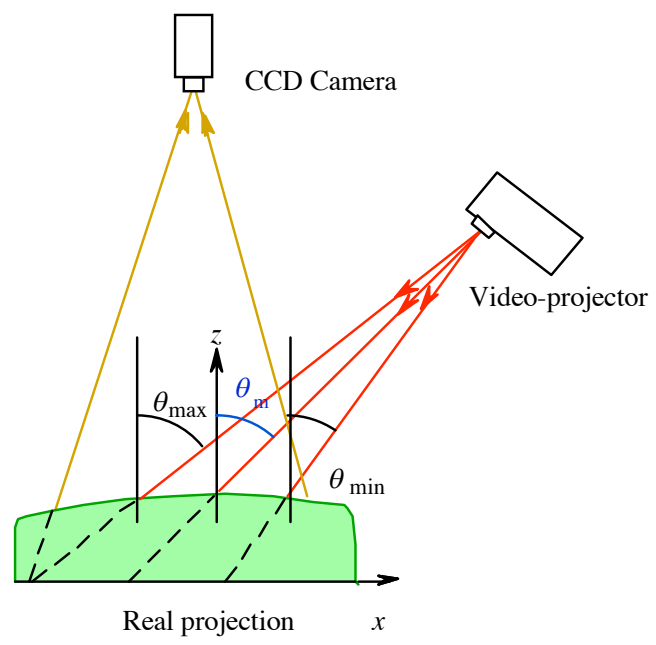

Figure 2

The knowledge of the phase allows to calculate the height $z$ from the equation (2). But the measure of the $\square_{\mathrm{m}}$ is difficult to do, the measurement incertainty is important so a calibration is required. One centimeter shifting of the reference surface measured with a displacement gauge allows a calibration and the calculation of the mean sensitivity of the set up. The students are faced to the fact that the sensitivity is nonuniform. They have to explain this, noticing that the projection angle varies between two values $\square_{\text {min }}$ and $\square_{\max }$. From the mean sensitivity value, the mean angle $\square_{\mathrm{m}}$ is deduced.

The axial resolution (along the $z$ axe) is deduced from the sensitivity and the resolution of the phase measurement. Knowing that the lateral resolution is equal to one pixel (at the object plane level) for the temporal phase-stepping, the pixel size in the object space is simply measured by the students by aiming at a ruler.

Finally the students have to measure an unknown spheric surface to determine its radius of curvature with its uncertainty.

In this experiment, the students'goal is to mesure a surface profile. To manage correctly this measurement, they have to analyse the work on the whole, to take the set up geometry into account, to evaluate the measurement uncertainties and try to minimize them. This lab work also pushes students to understand the importance of the configuration parameter choice in order to obtain the best results and to investigate the errors that have to be avoided. 